\title{
LINE FORMATION IN MOVING ATMOSPHERES
}

by

W. Kalkofen

Smithsonian Astrophysical observatory

and

Harvard College observatory

Cambridge, Massachusetts

\section{ABSTRACT}

We discuss an integral equation method that permits the calculation of the line source functions and of the emergent profiles in finite and semiinfinite atmospheres with macroscopic motion normal to the surface. Solutions are presented for a semiinfinite atmosphere with a temperature rise in the outward direction and with a flow that decays with increasing depth. The computed profiles have the form of $\mathrm{P}$ Cygni lines.

Key words: line formation, moving atmospheres, line-profile computation.

\section{INTRODUCTION}

Several authors have obtained numerical solutions of the equations of statistical equilibrium and radiative transfer. Magnan (1968) has employed the Monte Carlo method to determine the source function in a model of a planetary nebula; Hummer and Rybicki (1968) have solved a similar problem by means of differential equations with the Riccati transformation; and Kulander (1968) has used differential equations with the Eddington approximation in the solution of the equation of transfer for atmospheres with simple velocity fields. We describe here an integral equation method and apply it in the calculation of the source function in a model of an extended atmosphere. 
The various methods have certain limitations. In the Monte Carlo method the history of many photons is computed. Since the accuracy of the solution increases only slowly with the number of photons considered, the desired accuracy must be balanced against the thickness of the medium. In practice, this method is useful only for media with moderate optical thickness. However, the geometry of the medium and the physical processes taken into account may be quite complicated. Thus, incomplete redistribution of the photons over the line can be treated relatively easily. In the Riccati method the integration steps must be comparable with the shortest photon mean free path. Therefore, this method is also restricted to media with relatively small optical thickness. Kulander's technique permits the calculation of the source function in atmospheres with large optical thickness. But the use of the Eddington approximation in flow problems in which the Doppler shift in the normal direction may be large whereas the horizontal shift is zero can lead to large errors. The method is therefore suitable only for exploratory calculations.

The integral equation method is formulated for the case of a plane-parallel medium that may be finite or semi-finite and that has arbitrary flow along the normal to the surface. Anistropic microturbulence can be taken into account if it can be expressed in terms of normal and horizontal components. Complete frequency and angle redistribution of the photons is assumed so that the line component of: the source function is frequencyindependent and isotropic.

The conditions that we have imposed in the formulation of the integral equation method will frequently be realized approximately in stellar atmospheres. Thus, for lines we may usually assume that the atmosphere is plane-parallel over regions whose horizontal extent is large compared with the thermalization length. The gas flow will usually be directed along the normal to the surface. Othef flow patterns could, in principle, be treated by the same method. But differential motion within horizontal layers could not be dealt with easily. In general, however, the Doppler shift due to horizontal differential velocities that might occur, for example, in a uniform expansion should be small over a thermalization length. One may then compute the source function for a vanishing horizontal. velocity gradient but must take its effect into account in the calculation of the emergent flux. 
The requirement of complete redistribution will usualily be nearly satisfied, as Magnan has shown for a planetary nebula. In this context we note that the case of a semi-infinite stellar atmosphere is less severe than that of a planetary nebula, where the intensity outside the line becomes quite small, whereas in a semi-infinite medium the background radiation sets a limit on the intensity. We may therefore assume that the line source function is virtually frequency-independent and isotropic.

Because of these limitations of the methods, one would solve transfer problems for finite media with a complicated geometry using the Monte Carlo method, with a differential equation technique such as that of Hummer and Rybicki or that of Feautrier (1964), which is described in detail by Cuny (1967), or with the integral equation method of Jones and Skumanich (1968). For finite or semi-infinite media with a plane-parallel geometry, one would use either our integral equation or Feautrier's differential equation method. If the transfer is characterized by incomplete redistribution, one would choose the differential equation; if a large number of frequency and angle points has to be taken into account because of high flow velocities, one would choose the integral equation method. If both complications are present, one could attempt to reformulate the equations in terms of the local frequency variable that has been used by Magnan in the Monte Carlo method. For typical problems arising in extended atmospheres, both Feautrier's technique and our integral equation method could be used successfully, but the latter might give somewhat better results.

\section{THE BASIC EQUAT,IONS}

We seek the solution of the simultaneous equations of statistical equilibrium and radiative transfer. Following Thomas (1957), we express the statistical equilibrium equation in the form

$$
J-S=\varepsilon(S-\tilde{B}),
$$

where $S$ is the frequency- and angle-independent line component of the source function, $J$ is the mean integrated intensity, $\varepsilon$ is the probability of true 
absorption, and $\tilde{\mathrm{B}}$ is the Planck function that depends, in general, on the electron temperature and on the radiation field exclusive of the line radiation. We write the equation of radiative transfer. as

$$
J(\tau)-S(\tau)=H\left(\tau, \tau^{\prime}\right) S\left(\tau^{\prime}\right)+C(\tau),
$$

where $C$ is the contribution of the background radiation to the line intensity $J$, and $H$ is an integral operator. In the special case of vanishing background radiation, zero flow velocity, and isotropic Doppler broadening, $H$ is related to the well-known $\Lambda$-operator.

In the integral equation method we combine equations (1) and (2) into a single equation for the line component $S$ of the source function. The resulting equation, in matrix form, is solved for $s$ by means of a matrix inversion,

$$
S=(\varepsilon-H)^{-1}(\varepsilon \tilde{B}+C) .
$$

This procedure, with perhaps minor alterations, is standard for the integral equation method. For depth- and frequency-dependent but angle-independent absorption profiles, this method was developed by Athay and Skumanich (1967). The new feature that we want to discuss is the calculation of the matrix operator $\mathrm{H}$ when the absorption profile of the line depends on depth, frequency, and angle so that the monochromatic $\Lambda$-operator cannot be used.

We assume that the gas in the atmosphere is moving with the velocity $q$ along the outward normal of the plane-parallel atmosphere; $q$ is an arbitrary function of the depth. The quantity in the transfer equation that is modified by the flow is the absorption profile. If the line is broadened by the Doppler effect and by damping, the profile is given by the voigt function,

$$
\phi_{\Delta \nu, \mu}(\tau)=\frac{1}{\sqrt{\pi} \Delta \nu_{D}} \frac{a}{\pi} \int_{-\infty}^{\infty} \frac{e^{-y^{2}} d y}{a^{2}+(v-y)^{2}},
$$

where a is the damping parameter, $\Delta \nu_{D}$ is the Doppler 
width due to thermal and random microturbulent motion, and $v$ is given by

$$
v=\frac{\Delta \nu-\left(\nu_{O} / c\right) q(\tau) \mu}{\Delta \nu_{D}(\tau, \mu)}
$$

The frequency displacement $\Delta \nu$ is measured in the rest frame of the atom and $\nu_{O}$ is the frequency at the line center. We note that the Doppler width depends on direction when the microturbulence is anisotropic.

An important property of the absorption profile $\phi$ is its symmetry with respect to the frequency displacement $\Delta \nu$ and the direction cosine $\mu$,

$$
\phi_{\Delta \nu, \mu}(\tau)=\phi_{-\Delta \nu,-\mu}(\tau)
$$

Because of this symmetry in $\phi$ it is convenient to define an average intensity $I$ :

$$
\overline{\mathrm{I}}_{\Delta \nu, \mu}(\tau)=\frac{1}{2}\left[\mathrm{I}_{\Delta \nu, \mu}^{+}(\tau)+\mathrm{I}_{-\Delta \nu,-\mu}^{-}(\tau)\right], \mu>0,(7)
$$

in which the intensity in the forward direction along the ray $(\Delta \nu, \mu)$ is combined with the intensity in the opposite direction along the ray $(-\Delta \nu,-\mu)$. The integral form of the transfer equation for $\bar{I}$ is given by the equation

$$
\bar{I}_{\Delta \nu, \mu}\left(\tau_{\Delta \nu, \mu}\right)=\int_{0}^{T_{\Delta \nu, \mu}} d t k(\tau \Delta \nu, \dot{\mu}, t) \mathcal{S}_{\Delta \nu, \mu}(t),
$$

where $\mathcal{8}$ is the total source function,

$$
\mathscr{J}_{\Delta \nu, \mu}(\tau)=\frac{k^{L}(\tau) \phi_{\Delta \nu, \mu}(\tau) S(\tau) k^{C} \cdot(\tau) B(\tau)}{k^{L}(\tau) \phi_{\Delta \nu, \mu}(\tau)+k^{C}(\tau)},
$$

with $k^{L}$ and $k^{C}$ the opacities of the line and of the background continuum, respectively, and $\mathrm{B}$ the source 
function of the background continuum, and where $\tau_{\Delta \nu, \mu}$ is the specific monochromatic optical distance along the ray $(\Delta \nu, \mu) ; T_{\Delta \nu, \mu}$ is the corresponding distance between the two boundaries if the medium has finite optical thickness.

The integral kernel $\mathrm{K}$ is given by the equation

$$
K(\tau, t)=\frac{1}{2} e^{-|\tau-t|} .
$$

The kernel has the following asymptotic behavior:

$$
K(\tau, t)=\delta(\tau, t)\left\{1+\frac{d^{2}}{d \tau^{2}}+\sigma^{\prime}\left(e^{-\tau}\right)+\sigma\left[e^{-(T-\tau)}\right]\right\},(1
$$

where $\delta$ is the Dirac $\delta$-function. At large distances from the boundaries of the medium, therefore, the kernel $\mathrm{K}$ becomes essentially the second-derivative operator. The mean integrated intensity $\mathrm{J}$ is defined by the expression

$$
J(\tau)=\int_{-\infty}^{\infty} d(\Delta \nu) \int_{0}^{1} d \mu \phi_{\Delta \nu, \mu}(\tau) \overline{\mathrm{I}}_{\Delta \nu, \mu}(\tau)
$$

In order to solve the equations numerically, we choose a discrete set of depth points and expand the total source function $y^{\prime}$ in terms of piecewise quadratic segments. Instead of the integral operator involving the kernel $\mathrm{K}$, we obtain for equation (8) the product of a matrix operator and the vector of the total source function $\& 1$. By combining equations $(8),(9)$, and (12) and regrouping terms, we finally obtain the transfer equation in the form of equation (2).

\section{SOLUTIONS}

Our aim is to study the influence of macroscopic flow on the line source function and on the emergent monochromatic flux, and hence to investigate the formation of "P Cygni lines." To keep the interpretation relatively simple, we have worked with a single model of a semi-infinite plane-parallel atmos- 


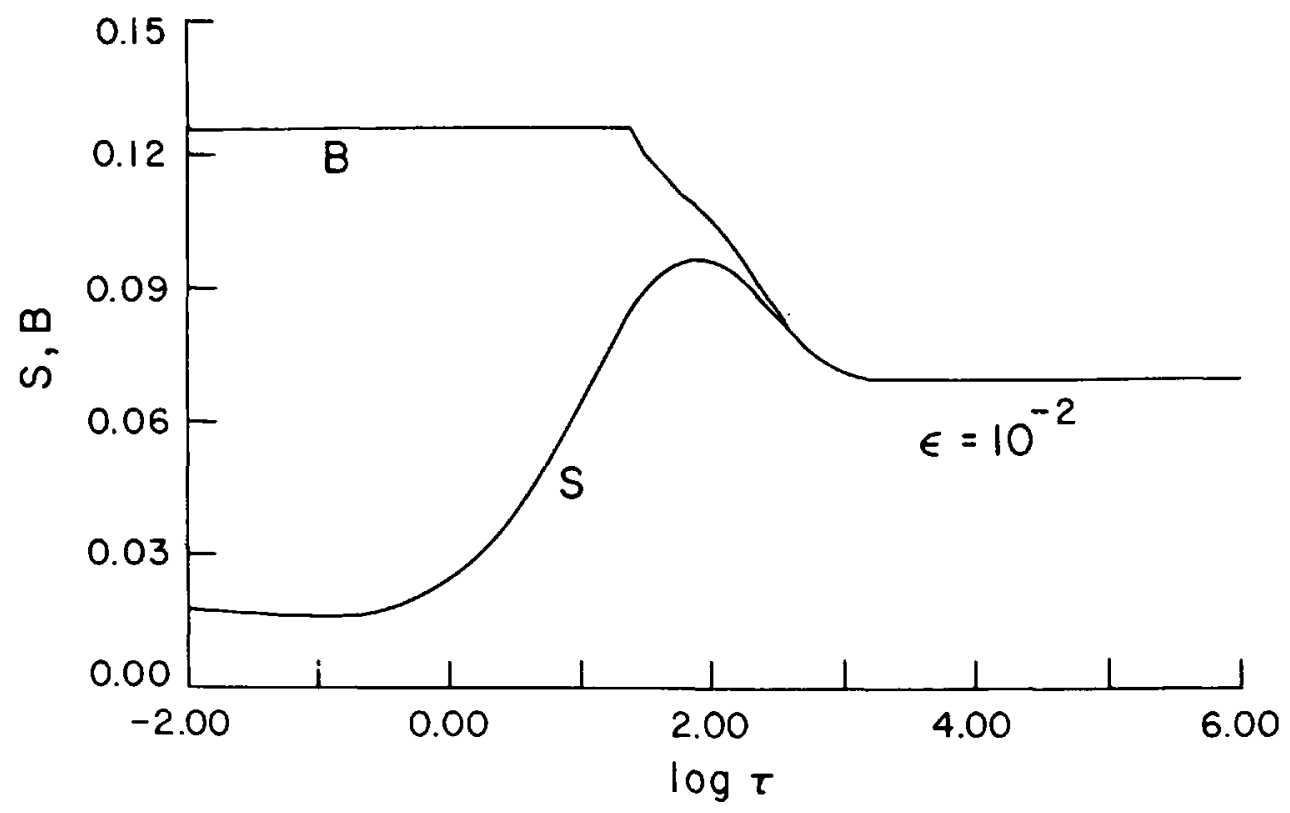

PLANCK FUNCTION AND SOURCE FUNCTION FOR

THE VELOCITY $v(\tau)=\frac{2}{1+\tau / 0.1}$

Figure 1.

phere with a temperature rising in the outer layers from $8000^{\circ} \mathrm{K}-10,000^{\circ} \mathrm{K}$. In this medium we compute a line source function with the frequency of the $\mathrm{H} \alpha$ line for $\varepsilon=10^{-2}, \mathrm{a}=0$, and constant thermal Doppler width. The function $\tilde{B}$ in equation (1) of statistical equilibrium is equal to the source function $B$ of the background continuum. The function $B(\tau)$, divided by $2 \mathrm{~h}_{\mathrm{v}}{ }^{3} / \mathrm{c}^{2}$, is plotted in Figures 1 and 3. The ratio of the opacity of the line at the center to that of the background continuum is constant with $\mathrm{k}^{\mathrm{L}} / \mathrm{k}^{\mathrm{C}}=10^{4}$. The velocities $\mathrm{q}(\tau)$ are given in units of the constant thermal Doppler width by the equation

$$
V(\tau)=\frac{V(0)}{1+\tau / T}
$$

where

$$
v=\frac{v_{0}}{C} \frac{g}{\Delta v_{D}}
$$

The optical depth $\tau$ is measured along the local line center.

In a preliminary investigation we have deter- 
ACCURACY OF THE ANGLE QUADRATURE

\begin{tabular}{|c|c|c|c|}
\hline $\begin{array}{l}\text { Angle } \\
\text { approximation }\end{array}$ & $S(0)$ & $\begin{array}{l}\text { Error (in p } \\
S(0.1)\end{array}$ & $\begin{array}{l}\text { percent) } \\
I(0.3)\end{array}$ \\
\hline 7 & 0.04 & 0.2 & 0.06 \\
\hline 6 & 0.1 & 0.5 & 0.16 \\
\hline 5 & 0.2 & 0.9 & 0.3 \\
\hline 4 & 0.3 & 1.4 & 0.6 \\
\hline 3 & 0.5 & 2.3 & 1.1 \\
\hline 2 & 1.3 & 4 & 2.5 \\
\hline 1 & 1.4 & 8 & 9 \\
\hline
\end{tabular}

mined the dependence of the computed source function, the emergent normal intensity, and the emergent monochromatic flux on the angle quadrature. For this purpose we have solved the equations with $\mathrm{V}(0)=2$ and $T=0.1$. This flow velocity produces a shift of the absorption profile by two Doppler widths at the surface, anci the velocity gradient is large only in the outermost layers. The source function has a minimum at a depth of $\tau=0.1$, and the minimum of the intensity is displaced in the violet direction by 0.3 thermal Doppler width. Table 1 gives the accuracy in percent of the solutions for. the first to the seventh angle approximations measured against the solution in the eighth approximation. All solutions were obtained with 63 frequency points in the range $(-4,4)$ of the frequency in Doppler units and 40 points in the depth range $\left(0,10^{6}\right)$. An accuracy of at least one percent in the quantities listed requires at least five discrete angle points. All subsequent solutions were therefore computed with the fifth angle points. All subsequent solutions were therefore computed with the fifth angle approximation. In the calculation of the monochromatic emergent flux, five angles did not give sufficiently accurate results. This quantity was therefore computed in the 24 th approximation. 
In order to investigate the effects of flow in a moving atmosphere, we have solved the equations for four cases of macroscopic flow and for the static atmosphere. For the flow problems we have chosen

$$
\mathrm{V}(0)=10 \text { and } \mathrm{T}=10,10^{2}, 10^{3}, 10^{4} \text {. }
$$

Thus the velocity is largest at the surface and decays essentially as $\tau^{-1}$. The gradient is large only near $\tau=T$. The computer plot of these velocities is given in Figure 2 .

We note that for $\varepsilon=10^{-2}$, the "thermalization length" of a Doppler-broadened line is $\tau_{0}=100$. The line that is formed in the atmosphere with the flow parameter $T=10^{4}$, therefore, sees only the constant flow velocity corresponding to 10 Doppler widths in the line formation region. Hence the source function should be identical with that of a static atmosphere. This is indeed the case, as can be seen from Figure 3, where the solutions for the stationary medium and for the atmosphere with

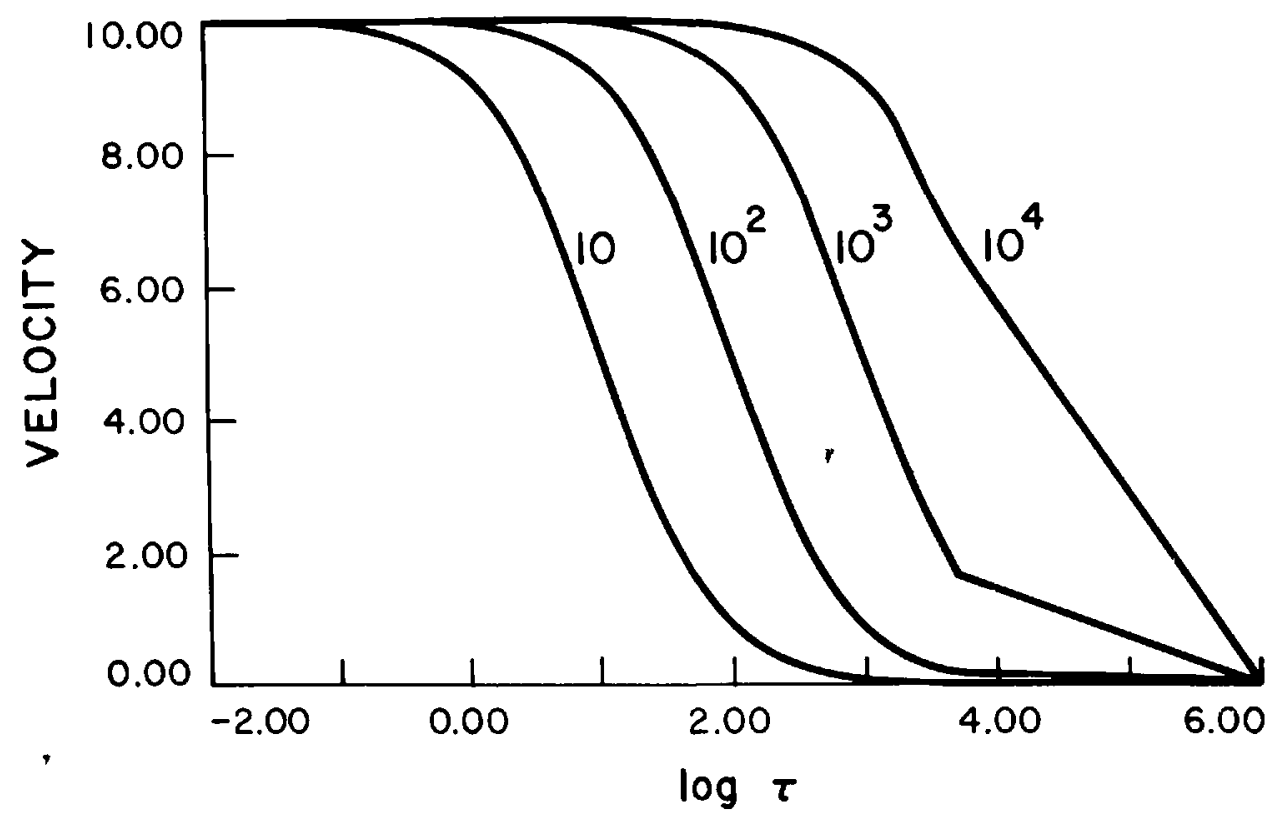

MACROSCOPIC VELOCITIES

$$
\begin{gathered}
v(\tau)=\frac{v(0)}{1+\tau / T}, v(0)=10, T=10,10^{2}, 10^{3}, 10^{4} \\
\text { Figure } 2 .
\end{gathered}
$$




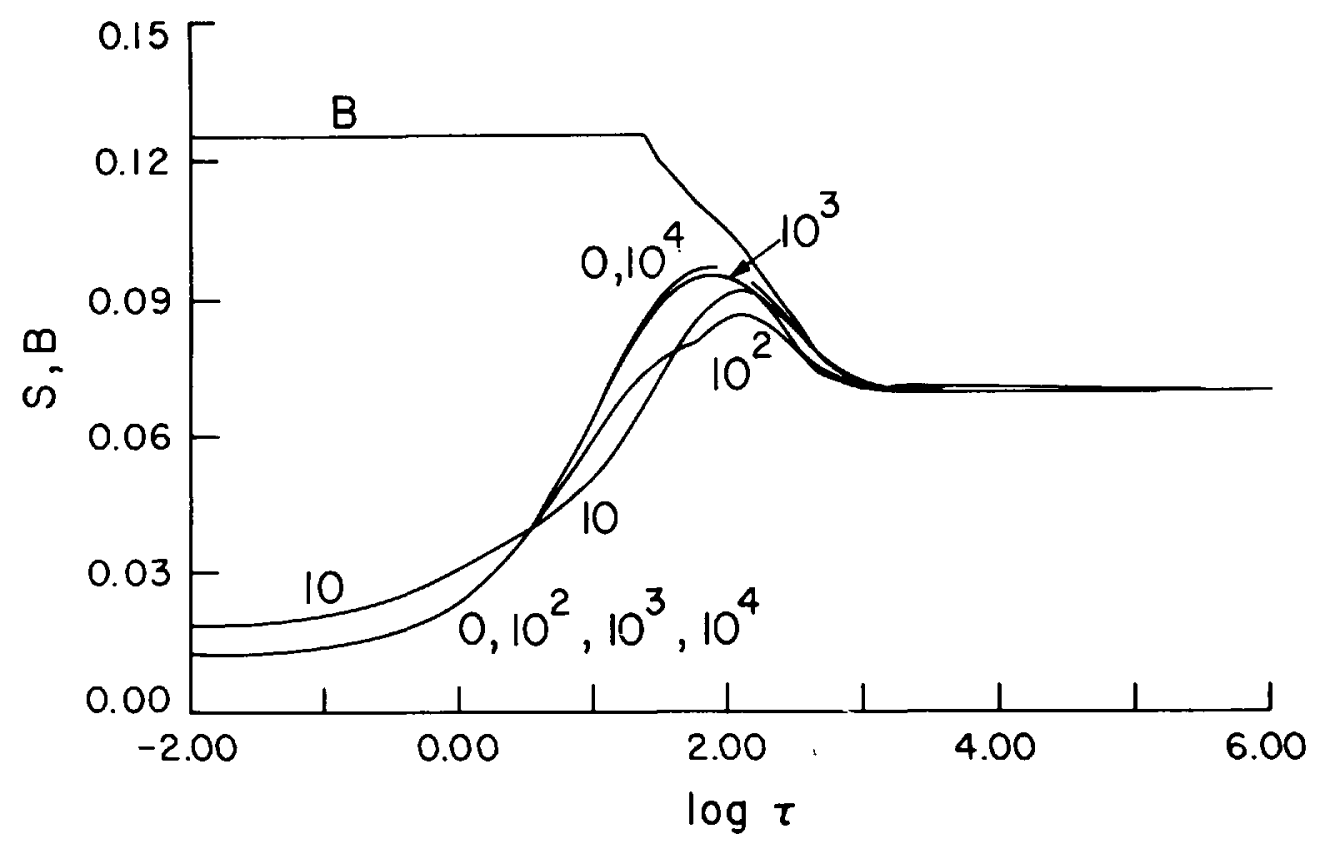

PLANCK FUNCTION AND LINE SOURCE FUNCTIONS FOR $\epsilon=10^{-2}$ AND $v(\tau)=\frac{10}{1+\tau / T}, T=10,10^{2}, 10^{3}, 10^{4}$. THE STATIC CASE IS LABELLED BY 0.

$$
\text { Figure } 3 \text {. }
$$

constant exparsion velocity in the significant surface layers coincide. For $T=10^{3}$, there is already a noticeable gradient in the macroscopic flow in the region of the atmosphere in which the line radiation reaches saturation. Because of the frequency shift of the line opacity, photons emitted near the maximum of $S$ can escape more easily than they can in the static case. The source function maximum is therefore reduced. The surface layers below $\tau=100$, however, still have a nearly constant flow velocity. The surface value of $S$ is therefore still virtually identical with that of the static solution. The same effects are seen in the source function for $T=100$, for which the velocity change is large in the relaxation region and small near the surface. Thus the value of the source function at its maximum is further reduced but its surface value is nearly unchanged. For $T=10$, the velocity gradient is small in the relaxation region and large near the surface. Near the source function maximum, the solution for $S$, therefore, moves back toward the static solution. But in the surface layers where $\mathrm{V}$ is varying rapidly, the line absorption coefficient is now large in a spectral range where the background radiation can exert a stronger 
influence on the line radiation. The surface value of $\mathrm{S}$ is consequently raised above that of the static case.

If the region in which the velocity gradient is large is pushed sufficiently close to the surface by reducing $T$ further, or if $\varepsilon$ is reduced significantly below $10^{-2}$, the source function can be forced by the background radiation to rise near the surface. This effect can, indeed, be seen in Figure 1, where $V(0)=2$ and $T=0.1$.

The most striking result of these calculations is the similarity in the solutions for a wide range of flows, which indicates that the source functions are not very sensitive to the Doppler shift. The emergent radiation field, however, is strongly influenced by the flow.

The emergent normal intensities are shown in Figure 4. For the static atmosphere the intensity is symmetric. For the flow parameter $\mathrm{T}=10^{4}$, the intensity is blue-shifted by 10 Doppler widths. The profile is nearly symmetric, only the slight enhancement of the red peak indicating the flow. For smaller values of the flow parameter $T$, the peaks on the low-frequency side of the line. center are more and more shifted to the red, the amplitudes having heights that correspond to the values of the maximum in the source functions. For $T=10$, the intensity minimum is shifted to $\Delta v=8.75$.

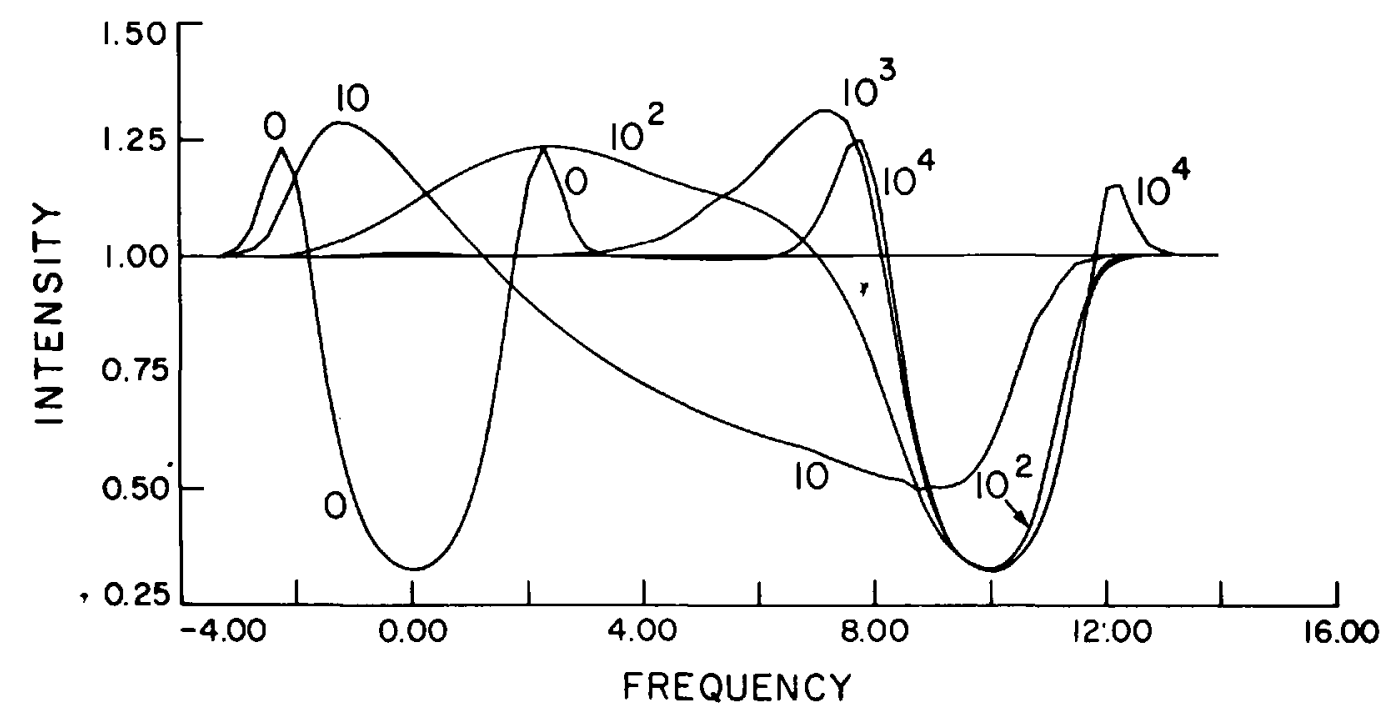

THE NORMAL, EMERGENT INTENSITY FOR $v(\tau)=\frac{v(0)}{1+\tau / T}$ WITH $v(0)=10$ AND $T=10,10^{2}, 10^{3}, 10^{4}$, AND FOR $v(0)=0$.

Figure 4 . 
The emergent monochromatic fluxes are plotted in Figure 5. Since the fluxes result from the weighted superposition of intensities for normal emergence, for which the frequency shift amounts to 10 Doppler widths, down to those for grazing emergence, for which the line is centered on $\Delta \nu=0$, the flux profiles are nonsymmetrical even for the uniform expansion given by the case of $T=10^{4}$. Only the static atmosphere produces a symmetrical flux profile. The flux curves for the flows with $T=10^{2}$ and $\mathrm{T}=10$ have peaks on the low-frequency side of the line profile. The condition for the appearance of such emission features is that the maximum of the source function fall into a region of the atmosphere for which the flow velocity is sufficiently small.

The minima of the fluxes are blue-shifted by $\Delta v=8.25$ thermal Doppler widths for the flow parameters $\mathrm{T}=10^{4}$ and $10^{3}$, by $\Delta \nu=7.75$ for $\mathrm{T}=10^{2}$, and by $\Delta v=4.5$ for $T=10$. The two maxima occur at $\Delta v=0.25$ for $T=10^{2}$ and at $\Delta \nu=1.75$ for $T=10$. The separation of the extrema is therefore $\Delta v=7.5$ Doppler widths for $T=10^{2}$ and $\Delta \nu=6.25$ for $T=10$. Thus, the blue shift of the central absorption feature is smaller than might have been expected on the basis of a rormal velocity displacement of $10^{4}$ Doppler widths, even when the outer layers expand uniformly as, in effect, they do for $\mathrm{T}=10^{4} ;$ and the separation of emission and absorption features

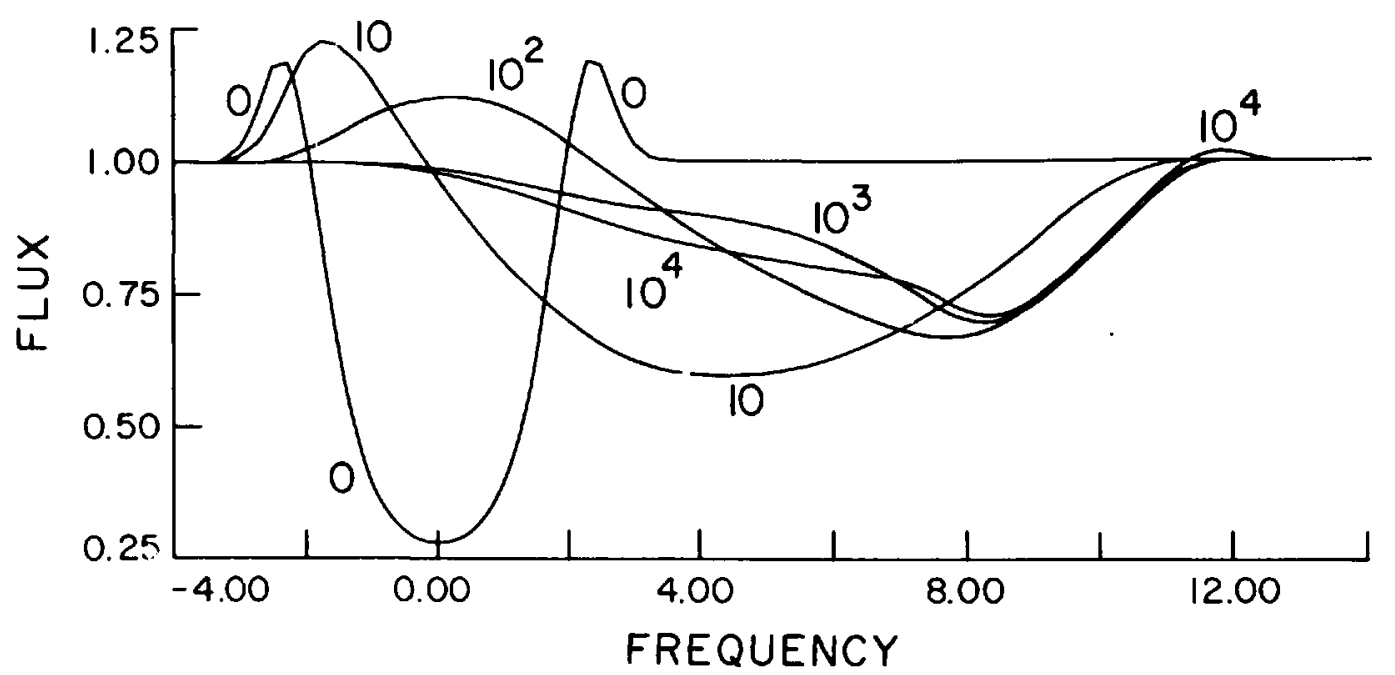

THE EMERGENT MONOCHROMATIC FLUX FOR $v(\tau)=\frac{10}{1+\tau / T}, T=10,10^{2}, 10^{3}, 10^{4}$, AND FOR $v=0$

Figure 5 . 
is not simply related to the flow velocity in the line formation region. The thermal Doppler width can be easily determined only in the line of the static atmosphere.

\section{CONCLUSIONS}

The profiles that we have computed have the general appearance of "P Cygni lines," from which they differ mainly in the relatively low emission intensities. The emission can be increased by means of a higher temperature rise in the outer layers of the star. Our model atmosphere was chosen more in accordance with the model calculations of Feautrier (1968) and of Auer and Mihalas (1969), who found temperature increases of $1000^{\circ}-2000^{\circ} \mathrm{K}$ for their models of early-type atmospheres in radiative and statistical equilibrium. Some mechanical mode of energy transport could give a much larger temperature rise as, for example, it does in the Sun. Such a temperature increase has been postulated for the supergiant $\alpha$ Cygni by Groth (1960), who furthermore placed the emission into an inwardfalling outer shell at a high temperature. Such an elaborate model atmosphere is clearly not necessary, as our calculations show. The only requirements for the appearance of $P$ Cygni lines are a temperature inversion in the atmosphere or envelope and flow in the outward direction.

It is a pleasure to thank Deane Peterson for discussions of the numerical method, Charles Whitney for useful comments on the interpretation of the results, and Lane Emerson for his help in computing and plotting with the aid of an electronic computer.

REFERENCES

Athay, R. G., and Skumanich, A. 1967, Ann. d'ap., 30,669 .

Aue $\bar{r}$. L. H., and Mihalas, D. 1969, Ap. J. (Letters), 156, I151.

Cuny, Y. 1967, Ann. d'ap., 30, 143.

Feautrier, P. 1964, C. R., 258, 3189; 1968, Ann. d'ap., 31, 257.

Groth, H. G. 1960, Z. Astrophys., 51, 231. 
Hummer, D. G., and Rybicki, G. B. 1968, Proc. Conf. on Resonance Lines in Astrophys., National Center for Atmospheric Research.

Jones, H. P., and Skumanich, A. 1968, Proc. Conf. on Resonance Lines in Astrophys., National Center for Atmospheric Research.

Kuhlander, J. L. 1968, J.Q.S.R.T., 8, 273.

Magnan, C. 1968, Ap. J. (Letters)', $\frac{2}{2}, 213$.

Thomas, R. N. 1957, Ap. J., 125, $26 \overline{0}$.

\section{DISCUSSION}

Underhill: How many levels did you include in your calculations?

Kalkofen: We included only two levels to show the principle of the problem.

Underhill: The observations do not show the

long tails which you have calculated.

Kalkofen: But there is also an increase in the line core, which is in agreement with the observation. If you apply the theory to the lines in the spectrum of $\varepsilon$ Ori the agreement is fairly good.

Underhill: The observations show only very weak $\mathrm{P}$ Cyg profiles in $\mathrm{H} \alpha$. 